\title{
Non-uniform dependence on initial data for the Camassa-Holm equation in the critical Besov space
}

\author{
Jinlu $\mathrm{Li}^{1 *}$, Xing $\mathrm{Wu}^{2 \dagger}$, Yanghai $\mathrm{Yu}^{3 \ddagger}$ and Weipeng $\mathrm{Zhu}^{4 \S}$ \\ ${ }^{1}$ School of Mathematics and Computer Sciences, Gannan Normal University, Ganzhou 341000, China \\ ${ }^{2}$ College of Information and Management Science, Henan Agricultural University, Zhengzhou 450002, China \\ 3 School of Mathematics and Statistics, Anhui Normal University, Wuhu 241002, China \\ ${ }^{4}$ School of Mathematics and Information Science, Guangzhou University, Guangzhou 510006, China
}

July 10, 2020

\begin{abstract}
Whether or not the data-to-solution map of the Cauchy problem for the CamassaHolm equation and Novikov equation in the critical Besov space $B_{2,1}^{3 / 2}(\mathbb{R})$ is not uniformly continuous remains open. In the paper, we aim at solving the open question left the previous works in $[27,28]$ and give a positive answer to this problem.
\end{abstract}

Keywords: Camassa-Holm (Novikov) equation, Non-uniform continuous dependence, Critical Besov spaces

MSC (2010): 35Q35; 35A01; 76W05

\section{Introduction}

In this paper, we consider the Cauchy problem for the well-known Camassa-Holm equation

$$
\begin{cases}u_{t}-u_{x x t}+3 u u_{x}=2 u_{x} u_{x x}+u u_{x x x}, & (x, t) \in \mathbb{R} \times \mathbb{R}^{+}, \\ u(x, t=0)=u_{0}, & x \in \mathbb{R} .\end{cases}
$$

$(\mathrm{CH})$ was firstly proposed in the context of hereditary symmetries studied in [18] and then was derived explicitly as a water wave equation by Camassa-Holm [4]. (CH) is completely integrable $[4,7]$ with a bi-Hamiltonian structure $[6,18]$ and infinitely many conservation laws $[4,18]$. Also,

\footnotetext{
*E-mail: lijinlu@gnnu.edu.cn

†E-mail: ny2008wx@163.com

$\ddagger$ E-mail: yuyanghai214@sina.com(Corresponding author)

$\S$ E-mail: mathzwp2010@163.com
} 
it admits exact peaked soliton solutions (peakons) of the form $c e^{-|x-c t|}$ with $c>0$, which are orbitally stable [15] and models wave breaking (i.e., the solution remains bounded, while its slope becomes unbounded in finite time $[5,10,11]$. It is worth mentioning that the peaked solitons present the characteristic for the travelling water waves of greatest height and largest amplitude and arise as solutions to the free-boundary problem for incompressible Euler equations over a flat bed, see Refs. $[8,12,13,30]$ for the details. Because of the mentioned interesting and remarkable features, the $\mathrm{CH}$ equation has attracted much attention as a class of integrable shallow water wave equations in recent twenty years. Its systematic mathematical study was initiated in a series of papers by Constantin and Escher, see [9-13]. We can refer the readers to see the global strong solutions in $[5,9,10]$ and finite time blow-up strong solutions in [5, 9-11] to $(\mathrm{CH})$, the existence and uniqueness of global weak solutions in [14,31], the global conservative solutions [2] and global dissipative solutions [3] in $H^{1}(\mathbb{R})$.

After the phenomenon of non-uniform continuity for some dispersive equations was studied by Kenig et al. [24], the issue of non-uniform dependence on the initial data has been a fascinating object of research in the recent past. Naturally, we may wonder which regularity assumptions are relevant for the initial data $u_{0}$ such that the Cauchy problem to $(\mathrm{CH})$ is not uniform dependence on initial data, namely, the dependence of solution on the initial data associated with this equation is not uniformly continuous. Himonas-Misiołek [23] obtained the first result on the non-uniform dependence for $(\mathrm{CH})$ in $H^{s}(\mathbb{T})$ with $s \geq 2$ using explicitly constructed travelling wave solutions, which was sharpened to $s>\frac{3}{2}$ by Himonas-Kenig [20] on the real-line and Himonas-KenigMisiołek [21] on the circle. Danchin [16,17] proved the local existence and uniqueness of strong solutions to $(\mathrm{CH})$ with initial data in $B_{p, r}^{s}$ if $(p, r) \in[1, \infty] \times[1, \infty), s>\max \left\{1+\frac{1}{p}, \frac{3}{2}\right\}$ and $B_{2,1}^{3 / 2}$. Li-Yin [25] proved that the continuity of the solution map of $(\mathrm{CH})$ with respect to the initial data. Guo et al. [19] established the ill-posedness of $(\mathrm{CH})$ in $H^{3 / 2}$ and in $B_{2, r}^{3 / 2}$ with $r \in(1, \infty)$ by proving the norm inflation. In our recent paper [27], we proved that the non-uniform dependence on initial data for $(\mathrm{CH})$ under the framework of Besov spaces $B_{p, r}^{s}$ for $s>\max \left\{1+\frac{1}{p}, \frac{3}{2}\right\}$. However, whether or not the data-to-solution map of the Cauchy problem for $(\mathrm{CH})$ in the critical Besov spaces $B_{2,1}^{3 / 2}(\mathbb{R})$ is not uniformly continuous remains open. We aim at giving a positive answer to this question in this paper.

Before stating our main result, we transform $(\mathrm{CH})$ equivalently into the following nonlinear transport type equation

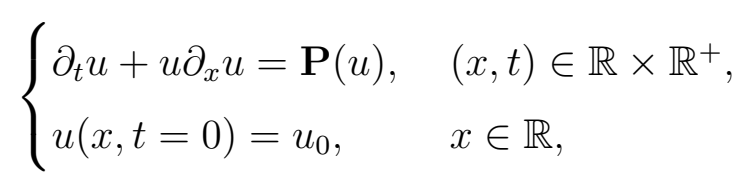

where

$$
\mathbf{P}(u)=P(D)\left(u^{2}+\frac{1}{2}\left(\partial_{x} u\right)^{2}\right) \quad \text { with } \quad P(D)=-\partial_{x}\left(1-\partial_{x}^{2}\right)^{-1}
$$

Our main result is stated as follows. 
Theorem 1.1 The solution map $u_{0} \rightarrow \mathbf{S}_{t}\left(u_{0}\right)$ of the Cauchy problem (1.1)-(1.2) is not uniformly continuous from any bounded subset in $B_{2,1}^{\frac{3}{2}}$ into $\mathcal{C}\left([0, T] ; B_{2,1}^{\frac{3}{2}}\right)$. More precisely, there exists two sequences of solutions $\mathbf{S}_{t}\left(f_{n}+g_{n}\right)$ and $\mathbf{S}_{t}\left(f_{n}\right)$ such that

$$
\left\|f_{n}\right\|_{B_{2,1}^{\frac{3}{2}}} \lesssim 1 \quad \text { and } \quad \lim _{n \rightarrow \infty}\left\|g_{n}\right\|_{B_{2,1}^{\frac{3}{2}}}=0
$$

but

$$
\liminf _{n \rightarrow \infty}\left\|\mathbf{S}_{t}\left(f_{n}+g_{n}\right)-\mathbf{S}_{t}\left(f_{n}\right)\right\|_{B_{2,1}^{\frac{3}{2}}} \gtrsim t, \quad \forall t \in\left[0, T_{0}\right]
$$

with small time $T_{0}$.

Remark 1.1 The method we used in [27] does not work for the critical index $s=\frac{3}{2}$ due to technical difficulty which mainly lies in the transport equation theory forbids the estimate of solution in $B_{2,1}^{1 / 2}$. We prove Theorem 1.1 by utilizing new method and generalize the previous result [27] to the critical case.

Remark 1.2 The method we used in proving the Theorem 1.1 can be applied equally well to other related systems, such as the following Novikov equation

$$
\left\{\begin{array}{l}
u_{t}+u^{2} u_{x}=\mathbf{Q}(u), \\
u(x, t=0)=u_{0},
\end{array}\right.
$$

where

$$
\mathbf{Q}(u)=-\left(1-\partial_{x}^{2}\right)^{-1}\left(\frac{1}{2} u_{x}^{3}+\partial_{x}\left(\frac{3}{2} u u_{x}^{2}+u^{3}\right)\right)
$$

Then we have the following

Theorem 1.2 The solution map $u_{0} \rightarrow \mathbf{S}_{t}\left(u_{0}\right)$ of the Cauchy problem (1.3)-(1.4) is not uniformly continuous from any bounded subset in $B_{2,1}^{\frac{3}{2}}$ into $\mathcal{C}\left([0, T] ; B_{2,1}^{\frac{3}{2}}\right)$. More precisely, there exists two sequences of solutions $\mathbf{S}_{t}\left(f_{n}+h_{n}\right)$ and $\mathbf{S}_{t}\left(f_{n}\right)$ such that

$$
\left\|f_{n}\right\|_{B_{2,1}^{\frac{3}{2}}} \lesssim 1 \quad \text { and } \quad \lim _{n \rightarrow \infty}\left\|h_{n}\right\|_{B_{2,1}^{\frac{3}{2}}}=0
$$

but

$$
\liminf _{n \rightarrow \infty}\left\|\mathbf{S}_{t}\left(f_{n}+h_{n}\right)-\mathbf{S}_{t}\left(f_{n}\right)\right\|_{B_{2,1}^{\frac{3}{2}}} \gtrsim t, \quad \forall t \in\left[0, T_{0}\right]
$$

with small time $T_{0}$.

Organization of our paper. In Section 2, we list some notations and known results which will be used in the sequel. In Section 3, we present the local well-posedness result and establish some technical Propositions. In Section 4, we prove our main theorem by adopting the strategies used 
in [27]. Here we should point out the new difficulty when dealing the critical case lies in the lack of the estimate of solution in $B_{2,1}^{\frac{1}{2}}$. To overcome this, we decompose the solution map as

$$
\mathbf{S}_{t}\left(u_{0}^{n}\right)=\mathbf{S}_{t}\left(u_{0}^{n}\right)-u_{0}^{n}-t \mathbf{v}_{0}\left(u_{0}^{n}\right)+f_{n}+g_{n}+t\left(\mathbf{P}\left(u_{0}^{n}\right)-u_{0}^{n} \partial_{x} u_{0}^{n}\right)
$$

On one hand, $u_{0}^{n} \partial_{x} u_{0}^{n}$ brings us the term $g_{n} \partial_{x} f_{n}$ which plays an essential role since it would not small when $n$ is large enough; On the other hand, $\mathbf{S}_{t}\left(u_{0}^{n}\right)-u_{0}^{n}-t \mathbf{v}_{0}\left(u_{0}^{n}\right)$ promotes us to estimate the crucial quantity $\left\|\mathbf{S}_{t}\left(u_{0}^{n}\right)\right\|_{L^{\infty}}$ which can be controlled by $t\left\|u_{0}^{n}\right\|_{C^{0,1}}^{2}+\left\|u_{0}^{n}\right\|_{L^{\infty}}$ instead of the norm $\left\|\mathbf{S}_{t}\left(u_{0}^{n}\right)\right\|_{B_{2,1}^{\frac{1}{2}}}$. Based on the suitable choice of $f_{n}$ and $g_{n}$, we prove that the solution map is not uniformly continuous.

\section{Littlewood-Paley analysis}

We firstly introduce some notations which will be used throughout this paper.

The symbol $A \lesssim(\gtrsim) B$ means that there is a uniform positive constant $c$ independent of $A$ and $B$ such that $A \leq(\geq) c B$. Given a Banach space $X$, we denote its norm by $\|\cdot\|_{X}$. We use the simplified notation $\left\|\mathbf{f}_{1}, \cdots, \mathbf{f}_{n}\right\|_{X}=\left\|\mathbf{f}_{1}\right\|_{X}+\cdots+\left\|\mathbf{f}_{n}\right\|_{X}$ if without confusion. For all $f \in \mathcal{S}^{\prime}$, the Fourier transform $\mathcal{F} f$ (also denoted by $\hat{f}$ ) is defined by

$$
\mathcal{F} f(\xi)=\hat{f}(\xi)=\int_{\mathbb{R}} e^{-i x \xi} f(x) \mathrm{d} x \quad \text { for any } \xi \in \mathbb{R} .
$$

The inverse Fourier transform allows us to recover $u$ from $\hat{f}$ :

$$
f(x)=\mathcal{F}^{-1} \hat{f}(x)=\frac{1}{2 \pi} \int_{\mathbb{R}} e^{i x \xi} \hat{f}(\xi) \mathrm{d} \xi .
$$

Next, we will recall some facts about the Littlewood-Paley decomposition, the nonhomogeneous Besov spaces and their some useful properties (see [1] for more details).

There exists a couple of smooth functions $(\chi, \varphi)$ valued in $[0,1]$, such that $\chi$ is supported in the ball $\mathcal{B} \triangleq\left\{\xi \in \mathbb{R}:|\xi| \leq \frac{4}{3}\right\}$, and $\varphi$ is supported in the $\operatorname{ring} \mathcal{C} \triangleq\left\{\xi \in \mathbb{R}: \frac{3}{4} \leq|\xi| \leq \frac{8}{3}\right\}$. Moreover,

$$
\chi(\xi)+\sum_{j \geq 0} \varphi\left(2^{-j} \xi\right)=1 \quad \text { for any } \xi \in \mathbb{R} .
$$

For every $f \in \mathcal{S}^{\prime}(\mathbb{R})$, the inhomogeneous dyadic blocks $\Delta_{j}$ are defined as follows

$$
\Delta_{j} f= \begin{cases}0, & \text { if } j \leq-2 \\ \chi(D) f=\mathcal{F}^{-1}(\chi \mathcal{F} f), & \text { if } j=-1 \\ \varphi\left(2^{-j} D\right) f=\mathcal{F}^{-1}\left(\varphi\left(2^{-j} \cdot\right) \mathcal{F} f\right), & \text { if } j \geq 0\end{cases}
$$

In the inhomogeneous case, the following Littlewood-Paley decomposition makes sense

$$
f=\sum_{j \geq-1} \Delta_{j} f \quad \text { for any } f \in \mathcal{S}^{\prime}(\mathbb{R}) .
$$


Definition 2.1 (See [1]) Let $s \in \mathbb{R}$ and $(p, r) \in[1, \infty]^{2}$. The nonhomogeneous Besov space $B_{p, r}^{s}(\mathbb{R})$ is defined by

$$
B_{p, r}^{s}(\mathbb{R}):=\left\{f \in \mathcal{S}^{\prime}(\mathbb{R}):\left\|\left(2^{j s}\left\|\Delta_{j} f\right\|_{L^{p}(\mathbb{R})}\right)_{j \in \mathbb{Z}}\right\|_{\ell^{r}(\mathbb{Z})}<\infty\right\}
$$

Finally, we give some important properties which will be also often used throughout the paper.

Lemma 2.1 (See [1]) For $s>0$, then for any $u, v \in B_{2,1}^{s}(\mathbb{R}) \cap L^{\infty}(\mathbb{R})$, we have

$$
\|u v\|_{B_{2,1}^{s}(\mathbb{R})} \leq C\left(\|u\|_{B_{2,1}^{s}(\mathbb{R})}\|v\|_{L^{\infty}(\mathbb{R})}+\|v\|_{B_{2,1}^{s}(\mathbb{R})}\|u\|_{L^{\infty}(\mathbb{R})}\right) .
$$

In particular, we have the embedding $B_{2,1}^{1 / 2}(\mathbb{R}) \hookrightarrow L^{\infty}(\mathbb{R})$ and

$$
B_{p, q}^{s}(\mathbb{R}) \hookrightarrow B_{p, r}^{t}(\mathbb{R}) \quad \text { for } s>t \quad \text { or } \quad s=t, 1 \leq q \leq r \leq \infty
$$

Lemma 2.2 (Lemma 3.26 in [1]) Let $(p, r) \in[1, \infty]^{2}, s>1$ and $u_{0} \in B_{p, r}^{s}(\mathbb{R})$. Assume that $u \in L^{\infty}\left([0, T] ; B_{p, r}^{s}(\mathbb{R})\right)$ solves $(1.1)-(1.2)$. Then there exists a constant $C=C(s, p)$ and a universal constant $C^{\prime}$ such that for all $t \in[0, T]$, we have

$$
\begin{aligned}
& \|u(t)\|_{B_{p, r}^{s}(\mathbb{R})} \leq\left\|u_{0}\right\|_{B_{p, r}^{s}(\mathbb{R})} \exp \left\{C \int_{0}^{t}\|u(\tau)\|_{C^{0,1}(\mathbb{R})} \mathrm{d} \tau\right\}, \\
& \|u(t)\|_{C^{0,1}(\mathbb{R})} \leq\left\|u_{0}\right\|_{C^{0,1}(\mathbb{R})} \exp \left\{C^{\prime} \int_{0}^{t}\left\|\partial_{x} u(\tau)\right\|_{L^{\infty}(\mathbb{R})} \mathrm{d} \tau\right\} .
\end{aligned}
$$

Let us recall the local well-posedness result for $(\mathrm{CH})$ in the critical Besov spaces.

Lemma 2.3 (See [17]) For any initial data $u_{0}$ which belongs to

$$
B_{R}=\left\{\psi \in B_{2,1}^{\frac{3}{2}}:\|\psi\|_{B_{2,1}^{\frac{3}{2}}} \leq R\right\} \quad \text { for any } R>0 .
$$

Then there exists some $T=T\left(\left\|u_{0}\right\|_{B_{2,1}^{\frac{3}{2}}}\right)>0$ such that $(C H)$ has a unique solution $\mathbf{S}_{t}\left(u_{0}\right) \in$ $\mathcal{C}\left([0, T] ; B_{2,1}^{\frac{3}{2}}\right)$. Moreover, we have

$$
\left\|\mathbf{S}_{t}\left(u_{0}\right)\right\|_{B_{2,1}^{\frac{3}{2}}} \leq C\left\|u_{0}\right\|_{B_{2,1}^{\frac{3}{2}}}
$$

\section{The Key Estimations}

Firstly, we need to introduce smooth, radial cut-off functions to localize the frequency region. Precisely, let $\hat{\phi} \in \mathcal{C}_{0}^{\infty}(\mathbb{R})$ be an even, real-valued and non-negative function on $\mathbb{R}$ and satisfy

$$
\hat{\phi}(\xi)= \begin{cases}1, & \text { if }|\xi| \leq \frac{1}{4} \\ 0, & \text { if }|\xi| \geq \frac{1}{2}\end{cases}
$$

Next, we need to establish the following crucial lemmas which will be used later on. 
Lemma 3.1 Let $(p, r) \in[1, \infty] \times[1, \infty)$. We define the high frequency function $f_{n}$ and the low frequency functions $g_{n}, h_{n}$ as follows

$$
\begin{aligned}
f_{n} & =2^{-\frac{3}{2} n} \phi(x) \sin \left(\frac{17}{12} 2^{n} x\right) \\
g_{n} & =\frac{12}{17} 2^{-n} \phi(x) \quad \text { and } \\
h_{n} & =\frac{12}{17} 2^{-\frac{n}{2}} \phi(x), \quad n \gg 1 .
\end{aligned}
$$

Then for any $\sigma \in \mathbb{R}$, we have

$$
\begin{aligned}
& \left\|f_{n}\right\|_{L^{\infty}} \leq C 2^{-\frac{3}{2} n} \phi(0) \quad \text { and } \quad\left\|\partial_{x} f_{n}\right\|_{L^{\infty}} \leq C 2^{-\frac{n}{2}} \phi(0), \\
& \left\|g_{n}, \partial_{x} g_{n}\right\|_{L^{\infty}} \leq C 2^{-n} \phi(0) \quad \text { and } \quad\left\|h_{n}, \partial_{x} h_{n}\right\|_{L^{\infty}} \leq C 2^{-\frac{n}{2}} \phi(0), \\
& \left\|g_{n}\right\|_{B_{p, r}^{\sigma}} \leq C 2^{-(n+\sigma)}\|\phi\|_{L^{p}} \quad \text { and } \quad\left\|h_{n}\right\|_{B_{p, r}^{\sigma}} \leq C 2^{-\left(\frac{n}{2}+\sigma\right)}\|\phi\|_{L^{p}} \\
& \left\|f_{n}\right\|_{B_{p, r}^{\sigma}} \leq C 2^{\left(\sigma-\frac{3}{2}\right) n}\|\phi\|_{L^{p}}, \\
& \liminf _{n \rightarrow \infty}\left\|g_{n} \partial_{x} f_{n}\right\|_{B_{2, \infty}^{\frac{3}{2}}} \geq M_{1}, \\
& \liminf _{n \rightarrow \infty}\left\|h_{n}^{2} \partial_{x} f_{n}\right\|_{B_{2, \infty}^{\frac{3}{2}}} \geq M_{2},
\end{aligned}
$$

for some positive constants $C, M_{1}, M_{2}$.

Proof. Direct computations gives (3.5)-(3.6). Notice that

$$
\operatorname{supp} \hat{g}_{n} \subset\left\{\xi \in \mathbb{R}: 0 \leq|\xi| \leq \frac{1}{2}\right\}
$$

then, we have

$$
\widehat{\Delta_{j} g_{n}}=\varphi\left(2^{-j} \xi\right) \hat{g}_{n}(\xi) \equiv 0 \quad \text { for } \quad j \geq 0
$$

which implies

$$
\Delta_{j} g_{n} \equiv 0 \quad \text { for } \quad j \geq 0
$$

By the definitions of $g_{n}$ and the Besov space, we deduce that

$$
\left\|g_{n}\right\|_{B_{p, r}^{\sigma}}=\frac{12}{17} 2^{-(n+\sigma)}\left\|\Delta_{-1} \phi\right\|_{L^{p}} \leq \frac{12}{17} 2^{-(n+\sigma)}\|\phi\|_{L^{p}}
$$

Notice that

$$
\operatorname{supp} \widehat{h_{n}} \subset\left\{\xi \in \mathbb{R}: 0 \leq|\xi| \leq \frac{1}{2}\right\} \Rightarrow \operatorname{supp} \widehat{h_{n}^{2}} \subset\{\xi \in \mathbb{R}: 0 \leq|\xi| \leq 1\},
$$

then, we have

$$
\operatorname{supp} \widehat{h_{n}^{2} \partial_{x} f_{n}} \subset\left\{\xi \in \mathbb{R}: \frac{17}{12} 2^{n}-\frac{3}{2} \leq|\xi| \leq \frac{17}{12} 2^{n}+\frac{3}{2}\right\}
$$


which implies

$$
\Delta_{j}\left(h_{n}^{2} \partial_{x} f_{n}\right)= \begin{cases}h_{n}^{2} \partial_{x} f_{n}, & \text { if } j=n, \\ 0, & \text { if } j \neq n .\end{cases}
$$

By the definitions of $f_{n}$ and $h_{n}$, we obtain for some $\delta>0$

$$
\begin{aligned}
\left\|h_{n}^{2} \partial_{x} f_{n}\right\|_{B_{2, \infty}^{\frac{3}{2}}} & =2^{\frac{3}{2} n}\left\|\Delta_{n}\left(h_{n}^{2} \partial_{x} f_{n}\right)\right\|_{L^{2}}=2^{\frac{3}{2} n}\left\|h_{n}^{2} \partial_{x} f_{n}\right\|_{L^{2}} \\
& =\left\|\frac{12}{17} \phi^{3}(x) \cos \left(\frac{17}{12} 2^{n} x\right)+\left(\frac{12}{17}\right)^{2} 2^{-n} \phi^{2}(x) \partial_{x} \phi(x) \sin \left(\frac{17}{12} 2^{n} x\right)\right\|_{L^{2}} \\
& \geq \frac{12}{17}\left\|\phi^{3}(x) \cos \left(\frac{17}{12} 2^{n} x\right)\right\|_{L^{2}}-C 2^{-n} \\
& \geq \frac{1}{8} \cdot \frac{12}{17} \delta \phi^{3}(0)\left(\int_{0}^{\delta}\left|\cos \left(\frac{17}{12} 2^{n} x\right)\right|^{2} \mathrm{~d} x\right)^{1 / 2}-C 2^{-n} .
\end{aligned}
$$

We thus deduce that (3.10)(see Lemma 1 in [27] for more details). Following the same procedure of the Proof of Lemmas 1-3 in [27], we can prove (3.8)-(3.9) with suitable modification. Here we omit the details.

Now, we establish the estimate involving $\mathbf{S}_{t}\left(u_{0}\right)-u_{0}-t \mathbf{v}_{0}\left(u_{0}\right)$ which is crucial in proving Theorem 1.1.

Proposition 3.1 Assume that $\left\|u_{0}\right\|_{B_{2,1}^{\frac{3}{2}}} \lesssim 1$. Under the assumptions of Theorem 1.1, we have

$$
\left\|\mathbf{S}_{t}\left(u_{0}\right)-u_{0}-t \mathbf{v}_{0}\left(u_{0}\right)\right\|_{B_{2,1}^{\frac{3}{2}}} \leq C t^{2} \mathbf{E}\left(u_{0}\right)
$$

where we denote $\mathbf{v}_{0}\left(u_{0}\right):=\mathbf{P}\left(u_{0}\right)-u_{0} \partial_{x} u_{0}$ and

$$
\mathbf{E}\left(u_{0}\right):=1+\left\|u_{0}\right\|_{C^{0,1}}^{2}|| u_{0}\left\|_{B_{2,1}^{\frac{5}{2}}}+\right\| u_{0} \|_{L^{\infty}}\left(\left\|u_{0}\right\|_{B_{2,1}^{\frac{5}{2}}}+\left(\left\|u_{0}\right\|_{L^{\infty}}+\left\|u_{0}\right\|_{C^{0,1}}^{2}\right)\left\|u_{0}\right\|_{B_{2,1}^{\frac{7}{2}}}\right) .
$$

Proof. For simplicity, we denote $u(t)=\mathbf{S}_{t}\left(u_{0}\right)$. Firstly, according to Lemma 2.3, there exists a small time $T=T\left(\left\|u_{0}\right\|_{B_{2,1}^{\frac{3}{2}}}\right)$ such that the solution $u(t) \in \mathcal{C}\left([0, T] ; B_{2,1}^{\frac{3}{2}}\right)$, namely,

$$
\|u(t)\|_{L_{T}^{\infty} B_{2,1}^{\frac{3}{2}}} \leq C\left\|u_{0}\right\|_{B_{2,1}^{\frac{3}{2}}} \leq C
$$

Applying Lemma 2.2 to Eq.(1.1), we have for all $t \in[0, T]$ and $\gamma \geq \frac{3}{2}$

$$
\|u(t)\|_{L_{T}^{\infty} B_{2,1}^{\gamma}} \leq\left\|u_{0}\right\|_{B_{2,1}^{\gamma}} \exp \left(C \int_{0}^{T}\|u\|_{B_{2,1}^{\frac{3}{2}}} \mathrm{~d} \tau\right) \leq C\left\|u_{0}\right\|_{B_{2,1}^{\gamma}} .
$$

Set $\widetilde{u}=\mathbf{S}_{t}\left(u_{0}\right)-u_{0}$, then we deduce from Eq.(1.1) that

$$
\left\{\begin{array}{l}
\partial_{t} \widetilde{u}+\mathbf{S}_{t}\left(u_{0}\right) \partial_{x} \mathbf{S}_{t}\left(u_{0}\right)=\mathbf{P}\left(\mathbf{S}_{t}\left(u_{0}\right)\right) \\
\widetilde{u}_{0}=0
\end{array}\right.
$$


Then, we have

$$
\begin{aligned}
\|\widetilde{u}(t)\|_{L^{\infty}} & \leq \int_{0}^{t}\left\|\partial_{\tau} \widetilde{u}\right\|_{L^{\infty}} \mathrm{d} \tau \\
& \leq \int_{0}^{t}\left\|\mathbf{S}_{t}\left(u_{0}\right) \partial_{x} \mathbf{S}_{t}\left(u_{0}\right)\right\|_{L^{\infty}} \mathrm{d} \tau+\int_{0}^{t} \| \mathbf{P}\left(\mathbf{S}_{t}\left(u_{0}\right) \|_{L^{\infty}} \mathrm{d} \tau\right. \\
& \leq C \int_{0}^{t}\left\|\mathbf{S}_{t}\left(u_{0}\right), u_{0}\right\|_{C^{0,1}}^{2} \mathrm{~d} \tau \quad \text { by Lemma } 2.2 \\
& \leq C t\left\|u_{0}\right\|_{C^{0,1}}^{2}
\end{aligned}
$$

where we have used the estimate

$$
\| \mathbf{P}\left(\mathbf{S}_{t}\left(u_{0}\right)\left\|_{L^{\infty}} \leq C\right\| \mathbf{S}_{t}\left(u_{0}\right) \|_{C^{0,1}}^{2}\right.
$$

from the fact that $\left(1-\partial_{x}^{2}\right)^{-1} f=G * f$ with $G(x)=\frac{1}{2} e^{-|x|}$.

Note that $P(D)$ is a multiplier of degree -1 , by Lemma 2.1, we have for $\gamma \geq \frac{3}{2}$

$$
\begin{aligned}
\|\mathbf{P}(u)\|_{B_{2,1}^{\gamma}} & \leq C\left\|u^{2}+\frac{1}{2} u_{x}^{2}\right\|_{B_{2,1}^{\gamma-1}} \\
& \leq C\left\|u, u_{x}\right\|_{L^{\infty}}\left\|u, u_{x}\right\|_{B_{2,1}^{\gamma-1}} \\
& \leq C\|u\|_{B_{2,1}^{\frac{3}{2}}}\|u\|_{B_{2,1}^{\gamma}} .
\end{aligned}
$$

By Lemma 2.1, we obtain from (3.12) and (3.16) that

$$
\begin{aligned}
\left\|u(t)-u_{0}\right\|_{B_{2,1}^{\frac{3}{2}}} & \leq \int_{0}^{t}\left\|\partial_{\tau} u\right\|_{B_{2,1}^{\frac{3}{2}}} \mathrm{~d} \tau \\
& \leq \int_{0}^{t}\|\mathbf{P}(u)\|_{B_{2,1}^{\frac{3}{2}}} \mathrm{~d} \tau+\int_{0}^{t}\left\|u \partial_{x} u\right\|_{B_{2,1}^{\frac{3}{2}}} \mathrm{~d} \tau \\
& \leq C t\left(\|u\|_{B_{2,1}^{\frac{3}{2}}}^{2}+\|u\|_{L^{\infty}}\left\|u_{x}\right\|_{B_{2,1}^{\frac{3}{2}}}\right) \\
& \leq C t\left(\left\|u_{0}\right\|_{B_{2,1}^{\frac{3}{2}}}^{2}+\left(\left\|u_{0}\right\|_{L^{\infty}}+\left\|u_{0}\right\|_{C^{0,1}}^{2}\right)\left\|u_{0}\right\|_{B_{2,1}^{\frac{5}{2}}}\right) .
\end{aligned}
$$

Similarly, we obtain from (3.12), (3.13) and (3.16) that

$$
\begin{aligned}
\left\|u(t)-u_{0}\right\|_{B_{2,1}^{\frac{5}{2}}} & \leq \int_{0}^{t}\left\|\partial_{\tau} u\right\|_{B_{2,1}^{\frac{5}{2}}} \mathrm{~d} \tau \\
& \leq \int_{0}^{t}\|\mathbf{P}(u)\|_{B_{2,1}^{\frac{5}{2}}} \mathrm{~d} \tau+\int_{0}^{t}\left\|u \partial_{x} u\right\|_{B_{2,1}^{\frac{5}{2}}} \mathrm{~d} \tau \\
& \leq C t\left(\|u\|_{B_{2,1}^{\frac{3}{2}}}\|u\|_{B_{2,1}^{\frac{5}{2}}}+\|u\|_{L^{\infty}}\|u\|_{B_{2,1}^{\frac{7}{2}}}\right) \\
& \leq C t\left(\left\|u_{0}\right\|_{B_{2,1}^{\frac{3}{2}}}\left\|u_{0}\right\|_{B_{2,1}^{\frac{5}{2}}}+\left(\left\|u_{0}\right\|_{L^{\infty}}+\left\|u_{0}\right\|_{C^{0,1}}^{2}\right)\left\|u_{0}\right\|_{B_{2,1}^{\frac{7}{2}}}\right) .
\end{aligned}
$$


Using Lemma 2.1 again, we obtain that

$$
\begin{aligned}
& \left\|u(t)-u_{0}-t \mathbf{v}_{0}\left(u_{0}\right)\right\|_{B_{2,1}^{\frac{3}{2}}} \leq \int_{0}^{t}\left\|\partial_{\tau} u-\mathbf{v}_{0}\left(u_{0}\right)\right\|_{B_{2,1}^{\frac{3}{2}}} \mathrm{~d} \tau \\
& \leq \int_{0}^{t}\left\|\mathbf{P}(u)-\mathbf{P}\left(u_{0}\right)\right\|_{B_{2,1}^{\frac{3}{2}}} \mathrm{~d} \tau+\int_{0}^{t}\left\|u \partial_{x} u-u_{0} \partial_{x} u_{0}\right\|_{B_{2,1}^{\frac{3}{2}}} \mathrm{~d} \tau \\
& \lesssim \int_{0}^{t}\left\|u(\tau)-u_{0}\right\|_{B_{2,1}^{\frac{3}{2}}} \mathrm{~d} \tau+\int_{0}^{t}\left\|u(\tau)-u_{0}\right\|_{L^{\infty}}\|u(\tau)\|_{B_{2,1}^{\frac{5}{2}}} \mathrm{~d} \tau \\
& +\int_{0}^{t}\left\|u(\tau)-u_{0}\right\|_{B_{2,1}^{\frac{5}{2}}}\left\|u_{0}\right\|_{L^{\infty}} \mathrm{d} \tau \\
& \lesssim \int_{0}^{t}\left\|u(\tau)-u_{0}\right\|_{B_{2,1}^{\frac{3}{2}}} \mathrm{~d} \tau+\left\|u_{0}\right\|_{B_{2,1}^{\frac{5}{2}}} \int_{0}^{t}\left\|u(\tau)-u_{0}\right\|_{L^{\infty}} \mathrm{d} \tau \\
& +\left\|u_{0}\right\|_{L^{\infty}} \int_{0}^{t}\left\|u(\tau)-u_{0}\right\|_{B_{2,1}^{\frac{5}{2}}} \mathrm{~d} \tau
\end{aligned}
$$

where we have used

$$
\begin{aligned}
\left\|\mathbf{P}(u)-\mathbf{P}\left(u_{0}\right)\right\|_{B_{2,1}^{\frac{3}{2}}} & \leq C\left\|\left(u+u_{0}\right)\left(u-u_{0}\right)+\frac{1}{2} \partial_{x}\left(u+u_{0}\right) \partial_{x}\left(u-u_{0}\right)\right\|_{B_{2,1}^{\frac{1}{2}}} \\
& \leq C\left\|u-u_{0}\right\|_{B_{2,1}^{\frac{3}{2}}}\left\|u+u_{0}\right\|_{B_{2,1}^{\frac{3}{2}}} \\
& \leq C\left\|u-u_{0}\right\|_{B_{2,1}^{\frac{3}{2}} .}
\end{aligned}
$$

Plugging (3.15)-(3.17) into (3.18) yields the desired result (3.11). Thus, we complete the proof of Proposition 3.1.

Also, the following estimate involving $\mathbf{S}_{t}\left(u_{0}\right)-u_{0}-t \mathbf{w}_{0}\left(u_{0}\right)$ is crucial in proving Theorem 1.2.

Proposition 3.2 Assume that $\left\|u_{0}\right\|_{B_{2,1}^{\frac{3}{2}}} \lesssim 1$. Under the assumptions of Theorem 1.2, we have

$$
\begin{aligned}
& \left\|\mathbf{S}_{t}\left(u_{0}\right)-u_{0}\right\|_{L^{\infty}} \leq C t\left\|u_{0}\right\|_{C^{0,1}}, \\
& \left\|\mathbf{S}_{t}\left(u_{0}\right)-u_{0}\right\|_{B_{2,1}^{\frac{3}{2}}} \leq C t\left(\left\|u_{0}\right\|_{B_{2,1}^{\frac{3}{2}}}^{3}+\left\|u_{0}\right\|_{C^{0,1}}^{2}\left\|u_{0}\right\|_{B_{2,1}^{\frac{5}{2}}}\right), \\
& \left\|\mathbf{S}_{t}\left(u_{0}\right)-u_{0}\right\|_{B_{2,1}^{\frac{5}{2}}} \leq C t\left(\left\|u_{0}\right\|_{B_{2,1}^{\frac{3}{2}}}^{2}\left\|u_{0}\right\|_{B_{2,1}^{\frac{5}{2}}}+\left\|u_{0}\right\|_{C^{0,1}}^{2}\left\|u_{0}\right\|_{B_{2,1}^{\frac{7}{2}}}\right), \\
& \left\|\mathbf{S}_{t}\left(u_{0}\right)-u_{0}-t \mathbf{w}_{0}\right\|_{B_{2,1}^{\frac{3}{2}}} \leq C t^{2} \mathbf{F}\left(u_{0}\right),
\end{aligned}
$$

where we denote $\mathbf{w}_{0}\left(u_{0}\right):=\mathbf{Q}\left(u_{0}\right)-u_{0}^{2} \partial_{x} u_{0}$ and

$$
\mathbf{F}\left(u_{0}\right):=1+\left\|u_{0}\right\|_{C^{0,1}}^{2}\left\|u_{0}\right\|_{B_{2,1}^{\frac{5}{2}}}+\left\|u_{0}\right\|_{C^{0,1}}^{4}\left\|u_{0}\right\|_{B_{2,1}^{\frac{7}{2}}} \cdot
$$

Proof. The proof follows the same manner from Proposition 3.1, we omit the details. 


\section{Non-uniform continuous dependence}

In this section, we prove Theorems 1.1 and 1.2 by using Propositions 3.1 and 3.2, respectively.

Proof of Theorem 1.1 We set $u_{0}^{n}=f_{n}+g_{n}$ and compare the solution $\mathbf{S}_{t}\left(u_{0}^{n}\right)$ and $\mathbf{S}_{t}\left(f_{n}\right)$. We obviously have

$$
\left\|u_{0}^{n}-f_{n}\right\|_{B_{2,1}^{\frac{3}{2}}}=\left\|g_{n}\right\|_{B_{2,1}^{\frac{3}{2}}} \leq C 2^{-n}
$$

which means that

$$
\lim _{n \rightarrow \infty}\left\|u_{0}^{n}-f_{n}\right\|_{B_{2,1}^{\frac{3}{2}}}=0
$$

From Lemma 3.1, one has

$$
\begin{aligned}
& \left\|u_{0}^{n}, f_{n}\right\|_{B_{2,1}^{\sigma}} \leq C 2^{\left(\sigma-\frac{3}{2}\right) n} \quad \text { for } \quad \sigma \geq \frac{3}{2} \\
& \left\|u_{0}^{n}, f_{n}\right\|_{L^{\infty}} \leq C 2^{-n} \quad \text { and } \quad\left\|\partial_{x} u_{0}^{n}, \partial_{x} f_{n}\right\|_{L^{\infty}} \leq C 2^{-\frac{n}{2}}
\end{aligned}
$$

which implies

$$
\mathbf{E}\left(u_{0}^{n}\right)+\mathbf{E}\left(f_{n}\right) \leq C .
$$

Notice that

$$
\begin{aligned}
& \mathbf{S}_{t}\left(u_{0}^{n}\right)=\underbrace{\mathbf{S}_{t}\left(u_{0}^{n}\right)-u_{0}^{n}-t \mathbf{v}_{0}\left(u_{0}^{n}\right)}_{=\mathbf{I}_{1}\left(\mathbf{v}_{0}\right)}+f_{n}+g_{n}+t\left(\mathbf{P}\left(u_{0}^{n}\right)-u_{0}^{n} \partial_{x} u_{0}^{n}\right) \\
& \mathbf{S}_{t}\left(f_{n}\right)=\underbrace{\mathbf{S}_{t}\left(f_{n}\right)-f_{n}-t \mathbf{v}_{0}\left(f_{n}\right)}_{=\mathbf{I}_{2}\left(\mathbf{v}_{0}\right)}+f_{n}+t\left(\mathbf{P}\left(f_{n}\right)-f_{n} \partial_{x} f_{n}\right) \text { and } \\
& u_{0}^{n} \partial_{x} u_{0}^{n}-f_{n} \partial_{x} f_{n}=g_{n} \partial_{x} f_{n}+u_{0}^{n} \partial_{x} g_{n},
\end{aligned}
$$

using the triangle inequality and Proposition 3.1, we deduce that

$$
\begin{aligned}
& \left\|\mathbf{S}_{t}\left(u_{0}^{n}\right)-\mathbf{S}_{t}\left(f_{n}\right)\right\|_{B_{2,1}^{\frac{3}{2}}} \\
= & \left\|\mathbf{I}_{1}\left(\mathbf{v}_{0}\right)-\mathbf{I}_{2}\left(\mathbf{v}_{0}\right)+g_{n}-t\left(g_{n} \partial_{x} f_{n}+u_{0}^{n} \partial_{x} g_{n}-\mathbf{P}\left(u_{0}^{n}\right)+\mathbf{P}\left(f_{n}\right)\right)\right\|_{B_{2,1}^{\frac{3}{2}}} \\
\geq & t\left\|g_{n} \partial_{x} f_{n}+u_{0}^{n} \partial_{x} g_{n}-\left(\mathbf{P}\left(u_{0}^{n}\right)-\mathbf{P}\left(f_{n}\right)\right)\right\|_{B_{2,1}^{\frac{3}{2}}}-\left\|\mathbf{I}_{1}\left(\mathbf{v}_{0}\right), \mathbf{I}_{2}\left(\mathbf{v}_{0}\right), g_{n}\right\|_{B_{2,1}^{\frac{3}{2}}} \\
\geq & t\left\|g_{n} \partial_{x} f_{n}\right\|_{B_{2,1}^{\frac{3}{2}}}-t\left\|u_{0}^{n} \partial_{x} g_{n}, \mathbf{P}\left(u_{0}^{n}\right)-\mathbf{P}\left(f_{n}\right)\right\|_{B_{2,1}^{\frac{3}{2}}}-C t^{2}-C 2^{-n} \\
\geq & t\left\|g_{n} \partial_{x} f_{n}\right\|_{B_{2,1}^{\frac{3}{2}}}-C t 2^{-n}-C t^{2}-C 2^{-n},
\end{aligned}
$$

where we have performed the following easy computations

$$
\begin{aligned}
& \left\|u_{0}^{n} \partial_{x} g_{n}\right\|_{B_{2,1}^{\frac{3}{2}}} \leq C\left\|u_{0}^{n}\right\|_{B_{2,1}^{\frac{3}{2}}}\left\|g_{n}\right\|_{B_{2,1}^{\frac{5}{2}}} \leq C 2^{-n} \\
& \left\|\mathbf{P}\left(u_{0}^{n}\right)-\mathbf{P}\left(f_{n}\right)\right\|_{B_{2,1}^{\frac{3}{2}}} \leq C\left\|g_{n}\right\|_{B_{2,1}^{\frac{3}{2}}}\left\|u_{0}^{n}+f_{n}\right\|_{B_{2,1}^{\frac{3}{2}}} \leq C 2^{-n} .
\end{aligned}
$$


Combining the fact from Lemma 3.1

$$
\liminf _{n \rightarrow \infty}\left\|g_{n} \partial_{x} f_{n}\right\|_{B_{2,1}^{\frac{3}{2}}} \gtrsim M_{1}
$$

then we deduce from (4.20) that

$$
\liminf _{n \rightarrow \infty}\left\|\mathbf{S}_{t}\left(f_{n}+g_{n}\right)-\mathbf{S}_{t}\left(f_{n}\right)\right\|_{B_{2,1}^{\frac{3}{2}}} \gtrsim t \quad \text { for } t \text { small enough. }
$$

This completes the proof of Theorem 1.1.

Proof of Theorem 1.2 We set $u_{0}^{n}=f_{n}+h_{n}$ and compare the solution $\mathbf{S}_{t}\left(u_{0}^{n}\right)$ and $\mathbf{S}_{t}\left(f_{n}\right)$. Obviously, we have

$$
\lim _{n \rightarrow \infty}\left\|u_{0}^{n}-f_{n}\right\|_{B_{2,1}^{\frac{3}{2}}}=\lim _{n \rightarrow \infty}\left\|h_{n}\right\|_{B_{2,1}^{\frac{3}{2}}}=0
$$

Lemma 3.1 tells us that

$$
\left\|u_{0}^{n}, f_{n}\right\|_{C^{0,1}} \leq C 2^{-\frac{n}{2}} \quad \text { and } \quad\left\|u_{0}^{n}, f_{n}\right\|_{B_{2,1}^{\sigma}} \leq C 2^{\left(\sigma-\frac{3}{2}\right) n} \quad \text { for } \quad \sigma \geq \frac{3}{2},
$$

which implies

$$
\mathbf{F}\left(u_{0}^{n}\right)+\mathbf{F}\left(f_{n}\right) \leq C .
$$

Using the triangle inequality and Proposition 3.2, we deduce that

$$
\begin{aligned}
& \left\|\mathbf{S}_{t}\left(u_{0}^{n}\right)-\mathbf{S}_{t}\left(f_{n}\right)\right\|_{B_{2,1}^{\frac{3}{2}}} \\
= & \left\|\mathbf{I}_{1}\left(\mathbf{w}_{0}\right)-\mathbf{I}_{2}\left(\mathbf{w}_{0}\right)+g_{n}-t\left(\left(u_{0}^{n}\right)^{2} \partial_{x} u_{0}^{n}-f_{n}^{2} \partial_{x} f_{n}-\mathbf{Q}\left(u_{0}^{n}\right)+\mathbf{Q}\left(f_{n}\right)\right)\right\|_{B_{2,1}^{\frac{3}{2}}} \\
\geq & t\left\|h_{n}^{2} \partial_{x} f_{n}\right\|_{B_{2,1}^{\frac{3}{2}}}-t\left\|2 f_{n} h_{n} \partial_{x} f_{n}+\left(u_{0}^{n}\right)^{2} \partial_{x} h_{n}+\mathbf{Q}\left(f_{n}\right)-\mathbf{Q}\left(u_{0}^{n}\right)\right\|_{B_{2,1}^{\frac{3}{2}}} \\
& -\left\|\mathbf{I}_{1}\left(\mathbf{w}_{0}\right), \mathbf{I}_{2}\left(\mathbf{w}_{0}\right), g_{n}\right\|_{B_{2,1}^{\frac{3}{2}}} \\
\geq & t\left\|h_{n}^{2} \partial_{x} f_{n}\right\|_{B_{2,1}^{\frac{3}{2}}}-C t 2^{-\frac{n}{2}}-C t^{2},
\end{aligned}
$$

where we have used that

$$
\left(u_{0}^{n}\right)^{2} \partial_{x} u_{0}^{n}-f_{n}^{2} \partial_{x} f_{n}=h_{n}^{2} \partial_{x} f_{n}+2 f_{n} h_{n} \partial_{x} f_{n}+\left(u_{0}^{n}\right)^{2} \partial_{x} h_{n}
$$

and

$$
\begin{aligned}
& \left\|\left(u_{0}^{n}\right)^{2} \partial_{x} h_{n}\right\|_{B_{2,1}^{\frac{3}{2}}} \leq C\left\|u_{0}^{n}\right\|_{B_{2,1}^{\frac{3}{2}}}^{2}\left\|h_{n}\right\|_{B_{2,1}^{\frac{5}{2}}} \leq C 2^{-\frac{n}{2}} \\
& \left\|\left.f_{n} h_{n} \partial_{x} f_{n}\right|_{B_{2,1}^{\frac{3}{2}}} \lesssim\right\| f_{n}\left\|_{L^{\infty}}\right\| h_{n}\left\|\left.\right|_{L^{\infty}}\right\| f_{n}\left\|_{B_{2,1}^{\frac{5}{2}}}+\right\| \partial_{x} f_{n}\left\|_{L^{\infty}}\right\| h_{n}\left\|_{B_{2,1}^{\frac{3}{2}}}\right\| f_{n} \|_{B_{2,1}^{\frac{3}{2}}} \leq C 2^{-n} .
\end{aligned}
$$

Combining the fact from Lemma 3.1

$$
\liminf _{n \rightarrow \infty}\left\|h_{n}^{2} \partial_{x} f_{n}\right\|_{B_{2,1}^{\frac{3}{2}}} \gtrsim M_{2}
$$


then we deduce from (4.21) that

$$
\liminf _{n \rightarrow \infty}\left\|\mathbf{S}_{t}\left(f_{n}+h_{n}\right)-\mathbf{S}_{t}\left(f_{n}\right)\right\|_{B_{2,1}^{\frac{3}{2}}} \gtrsim t \quad \text { for } t \text { small enough. }
$$

This completes the proof of Theorem 1.2.

Acknowledgements. J. Li is supported by the National Natural Science Foundation of China (Grant No.11801090). Y. Yu is supported by the Natural Science Foundation of Anhui Province (No.1908085QA05). W. Zhu is partially supported by the National Natural Science Foundation of China (Grant No.11901092) and Natural Science Foundation of Guangdong Province (No.2017A030310634).

\section{References}

[1] H. Bahouri, J. Y. Chemin, R. Danchin, Fourier Analysis and Nonlinear Partial Differential Equations, Grundlehren der Mathematischen Wissenschaften, Springer, Heidelberg, 2011.

[2] A. Bressan, A. Constantin, Global conservative solutions of the Camassa-Holm equation, Arch. Ration. Mech. Anal., 183 (2007), 215-239.

[3] A. Bressan, A. Constantin, Global dissipative solutions of the Camassa-Holm equation, Anal. Appl., 5 (2007), 1-27.

[4] R. Camassa, D. D. Holm, An integrable shallow water equation with peaked solitons, Phys. Rev. Lett., 71 (1993), 1661-1664.

[5] A. Constantin, Existence of permanent and breaking waves for a shallow water equation: ageometric approach, Ann. Inst. Fourier 50 (2000), 321-362.

[6] A. Constantin, The Hamiltonian structure of the Camassa-Holm equation, Exposition. Math., 15 (1997), 53-85.

[7] A. Constantin, On the scattering problem for the Camassa-Holm equation, R. Soc. Lond. Proc. Ser. A Math. Phys. Eng. Sci., 457 (2001), 953-970.

[8] A. Constantin, The trajectories of particles in Stokes waves, Invent. Math., 166 (2006), 523535.

[9] A. Constantin, J. Escher, Global existence and blow-up for a shallow water equation, Ann. Scuola Norm. Sup. Pisa Cl. Sci. (4), 26 (1998), 303-328.

[10] A. Constantin, J. Escher, Well-posedness, global existence, and blowup phenomena for a periodic quasi-linear hyperbolic equation, Comm. Pure Appl. Math., 51 (1998), 475-504.

[11] A. Constantin, J. Escher, Wave breaking for nonlinear nonlocal shallow water equations, Acta Math., 181 (1998), 229-243. 
[12] A. Constantin, J. Escher, Particle trajectories in solitary water waves, Bull. Amer. Math. Soc., 44 (2007), 423-431.

[13] A. Constantin, J. Escher, Analyticity of periodic traveling free surface water waves with vorticity, Ann. of Math., 173 (2011), 559-568.

[14] A. Constantin, L. Molinet, Global weak solutions for a shallow water equation, Comm. Math. Phys., 211 (2000), 45-61.

[15] A. Constantin, W. A. Strauss, Stability of peakons, Comm. Pure Appl. Math., 53 (2000), 603-610.

[16] R. Danchin, A few remarks on the Camassa-Holm equation. Differential Integral Equations, 14 (2001), 953-988.

[17] R. Danchin, A note on well-posedness for Camassa-Holm equation, J. Differential Equations, 192 (2003), 429-444.

[18] B. Fuchssteiner, A. Fokas, Symplectic structures, their Bäcklund transformation and hereditary symmetries, Phys. D, 4 (1981/82), 47-66.

[19] Z. Guo, X. Liu, M. Luc, Z. Yin, Ill-posedness of the Camassa-Holm and related equations in the critical space, J. Differential Equations, 266 (2019), 1698-1707.

[20] A. Himonas, C. Kenig, Non-uniform dependence on initial data for the CH equation on the line, Diff. Integral Eqns, 22 (2009), 201-224.

[21] A. Himonas, C. Kenig, Misiołek Non-uniform dependence for the periodic CH equation, Commun. Partial Diff. Eqns, 35 (2010), 1145-1162.

[22] A. Himonas, C. Holliman, The Cauchy problem for the Novikov equation, Nonlinearity, 25 (2012), 449-479.

[23] A. Himonas, G. Misiołek, High-frequency smooth solutions and well-posedness of the CamassaCHolm equation, Int. Math. Res. Not.,51 (2005), 3135-3151.

[24] C. Kenig, G. Ponce, L. Vega, On the ill-posedness of some canonical dispersive equations, Duke Math., 106 (2001) 617-633.

[25] J. Li, Z. Yin, Remarks on the well-posedness of Camassa-Holm type equations in Besov spaces, J. Differential Equations, 261 (2016), 6125-6143.

[26] J. Li, Z. Yin, Well-posedness and analytic solutions of the two-component Euler-Poincaré system, Monatsh. Math., 183 (2017), 509-537.

[27] J. Li, Y. Yu, W. Zhu, Non-uniform dependence on initial data for the Camassa-Holm equation in Besov spaces, J. Differential Equations, 269 (2020), 8686-8700.

[28] J. Li, M. Li, W. Zhu, Non-uniform dependence on initial data for the Novikov equation in Besov spaces, J. Math. Fluid Mech., https://doi.org/10.1007/s00021-020-00511-9 (2020). 
[29] G. Rodríguez-Blanco, On the Cauchy problem for the Camassa-Holm equation, Nonlinear Anal., 46 (2001), 309-327.

[30] J. F. Toland, Stokes waves, Topol. Methods Nonlinear Anal., 7 (1996), 1-48.

[31] Z. Xin and P. Zhang, On the weak solutions to a shallow water equation, Comm. Pure Appl. Math., 53 (2000), 1411-1433. 